Background: Rheumatoid Arthritis (RA) and Psoriatic arthritis (PsA) are both chronic, progressive inflammatory arthritis that can cause significant disability and morbidity. Depression in RA has been associated with higher levels of disease activity, pain, fatigue, work disability, lower treatment compliance and increased suicidal risk and mortality [1]. PsA patients suffer from psoriasis and joint involvement; hence have greater odds of depression by 2.1 times compared with RA [2].

Objectives: To compare the prevalence rates of depression and anxiety and its associated factors between RA and PsA patients in Hospital Putrajaya.

Methods: A cross sectional survey using the Hospital Anxiety and Depression Scale (HADS) questionnaire were distributed to 300 patients who attended rheumatology outpatient clinic from February - April 2019. The HADS was categorized into 3 groups based on their scores 0-7 (Normal); 8-10 (Borderline); and 11-21 (Abnormal). Data on patient demographics and components of disease assessment scores were recorded. Disease activity was assessed using DAS 28-CRP for all patients. Additional evaluation using Bath Ankylosing Spondylitis Disease Activity Score (BASDAI) and body surface area (BSA) were done for PsA patients. $P$ value of $<0.05$ was taken as significant.

Results: In total, 205 RA and 73 PsA patients were eligible for analysis. Majority of the patients were female, Malay and married for both groups. The mean age group for RA and PsA were $56.2 \pm 11.9$ years and $51.0 \pm 14.6$ years. The mean duration of disease for RA were $8 \pm 10$ years; while for PsA were $6 \pm 11$ years. The prevalence rates of depression and anxiety for RA were $8.3 \%$ and $13.7 \%$; and PsA were $9.6 \%$ and $17.8 \%$ respectively. Borderline scores for depression occurred in $16.1 \%$ of RA patients and $12.3 \%$ for PsA. Twenty percent of RA patients $(n=41)$ and twenty-four percent of PsA patients $(n=18)$ scored borderline for anxiety. The significant positive correlations with depression and anxiety in RA include high disease activity scores $(r=0.27 ; r=0.31)$, number of tender joints $(r=0.26 ; r=0.24)$ and pain $(r=0.29 ; r=0.27)$. Higher number of swollen joints significantly correlated with depression $(r=0.16)$ but not with anxiety. RA patients with Ischaemic Heart Disease (IHD) \pm heart failure have higher depression scores $(p<0.05)$. As for PsA group, high BASDAl score (anxiety: $r=0.34$ depression: $r=0.26)$ and psoriasis involving head and neck region $(p<0.05)$ were significant associated factors. Age was inversely correlated with anxiety in the PsA group.

Conclusion: There is higher prevalence of anxiety in both RA and PsA as compared to depression. Higher disease activity scores were associated with depression and anxiety in both RA and PsA with axial involvement.

References:

[1] Faith Matcham et al. "Are depression and anxiety associated with disease activity in rheumatoid arthritis? A prospective study" BMC Musculoskeletal Disorders (2016) 17:155.

[2] Sinnathurai et al. "Comorbids in psoriatic arthritis and rheumatoid arthritis". July 2018. Internal Medicine Journal.Available from https://doi.org/10.1111/ imj.14046

[3] RA Rahim et al. "Self-reported symptoms of depression, anxiety and stress among patients with Rheumatoid Arthritis in a Malaysian rheumatology centre - prevalence and correlates." Med J Malaysia Vol 73 No 4 August 2018

Disclosure of Interests: None declared

DOI: 10.1136/annrheumdis-2020-eular.5127

\section{FRI0078 \\ SERUM IRISIN LEVEL IN RHEUMATOID ARTHRITIS PATIENTS: ITS RELATIONSHIP TO DISEASE ACTIVITY AND CARDIOVASCULAR MANIFESTATIONS}

S. Soliman ${ }^{1}$, R. Gad ${ }^{1}$, T. Senosy ${ }^{2}$, A. Higazi ${ }^{3}$, R. Elshereef ${ }^{1} .{ }^{1}$ Faculty of Medicine, Minia University, Rheumatology \& Rehabilitation, Minia, Egypt; ${ }^{2}$ Faculty of Medicine, Minia University, Cardiology, Minia, Egypt; ${ }^{3}$ Faculty of Medicine, Minia University, Clinical Pathology, Minia, Egypt

Background: Active Rheumatoid Arthritis (RA) is associated with considerable changes in body composition, lipids, adipokines and insulin sensitivity. RA is an independent risk factor for CVD. The mechanisms leading to synovial inflammation are similar to those found in unstable atherosclerotic plaque. Irisin is a metabolic hormone and a novel adipomyokine related to insulin resistance and endothelial functions (1)

Objectives: To investigate the relationship between serum irisin levels, disease activity and cardiovascular risk in RA patients, and to test its performance in predicting subclinical atherosclerosis in RA patients.

Methods: 60 RA patients fulfilling the 2010 ACR/EULAR RA Classification Criteria and 30 healthy controls were recruited for serological testing of irisin levels. BMI was calculated. Waist/hip ratio was measured. RA disease activity was assessed by DAS28-ESR. Disability was assessed by HAQ-DI in its Arabic version. Serum ESR, CRP, glycated hemoglobin (HbA1c), lipid profile (serum level of cholesterol, triglyceride, HDL, LDL and cholesterol/ LDL ratio), insulin levels were measured in all patients and controls. Homeostasis Model Assessment of Insulin Resistance (HOMA-IR) was used to calculate insulin resistance.
Carotid intimal medial thickness (C-IMT), an indicator of atherosclerosis, was measured by carotid doppler ultrasonography. Echocardiography was performed to assess cardiac abnormalities. Our RA patients were classified twice first, according to cardiovascular abnormalities and second, according to cutoff values of DAS28.

Results: Serum irisin levels were significantly lower in RA patients $(9.84 \pm$ $10.56) \mathrm{ng} / \mathrm{ml}$ compared to controls $(20.48 \pm 13.82) \mathrm{ng} / \mathrm{ml}(p<0.001)$. BMl values were significantly higher in all patients than controls $(P=0.035)$, while waist/hip ratio in female patients only were significantly higher $(P=0.007)$. We found a negative correlation between serum irisin and DAS28-ESR $(r=-0.455$, P-value $0.005) \&$ HAQ-DI $(r=-0.309$, P-value 0.016$)$. There was a negative correlation between serum irisin level and parameters of cardiovascular risk including anthropometric measurements (BMI and waist/hip ratio), HOMA-IR ( $r=-0.371$, $\mathrm{p}=0.009)$ and C-IMT $(r=-0.511, p<0.001)$. No correlation could be detected between irisin and lipid profile. The frequency of cardiovascular (CV) involvement in RA patients was $45 \%$ (27 patients) (11.6\% with echocardiographic abnormalities and $40 \%$ having increased C-IMT). Patients with CV involvement showed lower serum irisin level, increased disease activity assessed by DAS28 and increased disease disability assessed by HAQ-DI with statistically significant difference $(P<0.001, P<0.05$ and $P<0.001$ respectively) Classifying the patients based on cut-off values of DAS28 into 3 groups (low disease activity, moderate and high disease activity), we found a statistically significant difference between the irisin levels of the 3 groups, being lowest among highly active patients $(P=0.014)$. $\mathrm{C}$-IMT values were significantly higher in highly active patients $(P=0.04)$. Assessing the biomarker's performance as an independent indicator of subclinical atherosclerosis in RA patients using ROC curve, it showed an excellent ability (AUC 0.8, P <0.001). As regarding its ability to differentiate patients with high disease activity, it showed a very good performance (AUC 0.73, $\mathrm{P}<0.001$ ).

Conclusion: In RA patients, serum irisin level was significantly lower and perform better than traditional yardsticks in identifying disease activity. It may act as an independent indicator of subclinical atherosclerosis in RA patients Serum irisin level may be responsible for increased cardiovascular risk in those patients.

\section{References:}

[1] Chen JQ, Huang YY, Gusdon AM, Qu S. Irisin: a new molecular marker and target in metabolic disorder. Lipids Health Dis. 2015 Jan 14; 14:2.

Disclosure of Interests: None declared

DOI: 10.1136/annrheumdis-2020-eular.2397

\section{FRI0079 CHARACTERISTICS OF DIFFICULT-TO-TREAT RHEUMATOID ARTHRITIS}

S. Takanashi ${ }^{1}$, Y. Kaneko ${ }^{1}$, T. Takeuchi ${ }^{1}{ }^{1}$ Keio University School of Medicine, Division of Rheumatology, Department of Internal Medicine, Tokyo, Japan

Background: Despite remarkable progress in therapy, not a few patients with rheumatoid arthritis (RA) have not achieved treatment target. Various factors can be ascribed to difficult-to-treat RA, however, little is known about their characteristics.

Objectives: To clarify characteristics of patients with difficult-to-treat RA in real-world.

Methods: We reviewed all consecutive RA patients in Keio University Hos pital between 2016 and 2017 and collected medical information. We defined patients in moderate disease activity and high disease activity according to disease activity score for 28 joints (DAS28) at the last visit despite more than one year treatment for RA as difficult-to-treat RA and analyzed their clinical characteristics.

Results: A total of 1693 patients with RA were enrolled in the analysis. The mean age at the last visit was 64 years old, female was $83 \%$, and the mean disease duration was 11.9 years. Rheumatoid factor and anti-cyclic citrullinated peptide were positive for $76 \%$ and $75 \%$ of the patients, respectively. The current treatment were conventional synthetic disease modifying anti-rheumatic drugs in $73 \%$, biologic agents or janus kinase (JAK) inhibitors in $57 \%$, and glucocorticoids in $13 \%$. Disease activity according to DAS28 was remission in $65 \%$, low disease activity in $21 \%$, and moderate/high disease activity in $14 \%$, which was defined as difficult-to-treat RA. Characteristics of difficult-to-treat RA were the mean age of 70 years old, female of $89 \%$, and the mean disease duration of 14.8 years. The current treatments were conventional synthetic disease modifying anti-rheumatic drugs alone in $40.7 \%$, biologic agents or JAK inhibitors in $55.8 \%$, and glucocorticoids in $29.0 \%$. The causes of difficult-to-treat RA were unresponsiveness to several biologic agents and/or JAK inhibitors in $22.9 \%$, comorbidities in $33.8 \%$, and personal reasons in $39.8 \%$ (costs in $35.9 \%$, low adherence in $4.3 \%$, concerns about possible adverse reaction of drugs in $54.3 \%$ and high patient global assessment in 5.4\%). Patient characteristics were sig nificantly different between the causes; age at RA onset (51 vs 61 vs 51 years, $p<0.001$ ), current age (65 vs 77 vs 66 years, $p<0.001$ ), estimated glomerular 